\title{
STATIC ANALYSIS OF FUNCTIONALLY GRADED PLATE USING NON- LINEAR CLASSICAL PLATE THEORY WITH VON-KARMAN STRAINS
}

\author{
S.J. SINGH* and S.P. HARSHA \\ Mechanical and Industrial Engineering Department \\ Indian Institute of Technology, Roorkee \\ Roorkee, Uttarakhand-247667, INDIA \\ E-mails: sim87.dme2015@iitr.ac.in; surajfme@iitr.ac.in
}

\begin{abstract}
The present study is based on the nonlinear bending analysis of an FGM plate with Von-Karman strain based on the non-linear classical plate theory (NLCPT) with in-plane displacement and moderate rotation. Non-linear bending analysis based on stresses and transverse deflections is then carried out for the plate for the complex solution obtained using an analytical method viz. Navier's method. The equations of motion and boundary conditions are obtained using the Principle of Minimum Potential Energy (PMPE) method and material property is graded in thickness direction according to simple power-law distribution in terms of volume fractions of the constituents. The effect of the span-to-thickness ratio and FGM exponent on the maximum central deflection and stresses are studied. The results show that the response is transitional with respect to ceramic and metal and the complex solution predicts the real behavior of stresses and deflections in the functionally graded plate. The functionally graded plate is found to be more effective for moderately thick and thick plates, which is inferred by a complex nature of the solution. For FGM plates, the transverse deflection is in-between to that of metal and ceramic rich plates. The complex nature of the solution also gives information about the stress distribution in the thickness direction.
\end{abstract}

Key words: functionally graded materials (FGM) plate, Von-Karman strain, nonlinear classical plate theory (NLCPT); Navier's method.

\section{Introduction}

Plates are the structural components that are initially straight and having a thickness much smaller than other dimensions. The transverse loading applied to the plate surface is supported by the bending moment, torsional moment, axial, transverse shear force and central shear force depending upon the type of plate in the application. In recent years, a new class of materials known as smart materials has been introduced in engineering applications. Functionally Graded Materials (FGM) are one of the smart materials introduced nowadays, in which material properties of two materials are graded in one particular direction resulting in corresponding changes in the properties of the material. The ultimate purpose of development of this advanced material is to explore quiescent applications in actual structures. FGMs may be incorporated in the form of a beam, plate, or shell as structural components and have great potential in applications where the operating conditions are severe, including spacecraft heat shields, heat exchanger tubes, biomedical implants, flywheels, and plasma facings for fusion reactors, etc. Functionally Graded Materials (FGMs) were substantially advanced in the early 1980's in Japan, where this new material concept was proposed to increase adhesion and minimize the thermal stresses in metallic-ceramic composites developed for reusable rocket engines [1]. Meanwhile, FGMs concepts have triggered worldwide research activity and are applied to metals, ceramics and organic composites to generate improved components with superior physical properties [2]. Depending on the application and the specific loading conditions, varying approaches can be followed to

\footnotetext{
${ }^{*}$ To whom correspondence should be addressed
} 
generate the structure gradients. A functionally graded material eliminates the sharp interfaces existing in a composite material where failure is initiated [3]. It replaces this sharp interface with a gradient interface which produces a smooth transition from one material to the next $[4,5]$. Functionally graded materials can withstand a very high thermal gradient, this makes it suitable for use in structures and space plane body, rocket engine component, etc. [6]. FGMs are used in energy conversion devices. They also provide a thermal barrier and are used as a protective coating on turbine blades in a gas turbine engine [7, 8]. Levy et al. [9] obtained solutions for a wide range of values of the loading parameter and the aspect ratio for the large deflections of rectangular plates using an asymptotic solution. Kant et al. [10] presented a model based on the refined Higher-order Shear Deformation Theory (HSDT) for laminated composite and sandwich plates and it was also assumed that the thickness coordinate is not constant, thus giving a more accurate result in comparison to other theories. Huang et al. [11] presented a generalized Fourier series solution for a moderately thick rectangular plate of variable thickness and arbitrary boundary conditions based on the linear First-order Shear Deformation Theory (FSDT). Liao et al. [12] studied the size effect on dynamic stability of functionally graded microbeams based on a modified couple stress theory using Hamilton's principle for deriving the equation of motion and the Differential Quadrature Method (DQM) for converting the governing differential equations into a linear system of Mathieu-Hill equations from which the boundary points on the unstable regions are determined by Bolotin's method. Size-dependent functionally graded Kirchhoff and Mindlin plate models [13], Reddy plate model [14], sinusoidal plate model [15] based on modified couple stress theory, and functionally graded new sinusoidal shear deformation theory [16] have been developed for vibration, bending and buckling analysis. The analysis was carried out by making use of Hamilton's principle for the equation of motion and solving it by Navier's approach. Reddy et al. [17] presented a paper on the static analysis of FGM plates using HSDT without enforcing zero transverse shear stress on the top and bottom surfaces of the plate, thus incorporated the transverse extensibility which accounts for the transverse effects and absence of shear correction factor. Kumar et al. [18] investigated the flexural response of FGM plates by utilizing different algebraic shear deformation theories and solved the resulting governing differential equations using Multiquadric radial basis function (MQRBF). Setoodeh et al. [19] formulated the DQM in conjunction with the introduced transformed weighing coefficients (TW-DQ) to solve geometrically nonlinear free vibration of FG-CNTRC quadrilateral plates. In the present study, the CPT together with von Karman's strain-displacement assumptions was employed to derive the nonlinear governing partial differential equations with geometric nonlinearity. Neves et al. addressed the analysis of functionally graded plates by collocation with radial basis functions, according to a sinusoidal shear deformation formulation [20],quasi-3D hyperbolic sine shear deformation theory [21], quasi-3D sinusoidal shear deformation formulation [22], quasi-3D higher-order shear deformation theory and a meshless technique [23]. Hassaine et al. [24] presented a theoretical formulation, of Navier solutions of rectangular plates based on a new higher order shear deformation model for the static response of functionally graded plates (FGPs). Kulkarni et al. [25] attempted to extend the inverse trigonometric shear deformation theory (ITSDT) for the static and buckling analysis of FGPs using an analytical solution.

It has been observed from the available literature that static and dynamic analysis of FGM plates based on different theories, viz. classical theories have been studied which analyzed stresses, deflection and vibration using different numerical and analytical solutions. In the present study, the CPT together with von Karman's strain-displacement assumptions have been employed to derive the nonlinear governing partial differential equations with geometric nonlinearity.

\section{Geometry}

The thin plate is a planar structure having the transverse dimension much smaller as compared to the length and width dimensions. Let us consider a stiff plate whose transverse dimensions are small as compared to the other two dimensions and, therefore, the plate is considered to be a two-dimensional lamina. Assume a mid-plane of the plate as a reference surface in the $x-y$ plane and the $z$ - axis along the transverse 
direction, with the $z$ coordinate positive upward, forming a right-handed coordinate system. The reference surface is at the distance of $\pm h / 2$ from both top and bottom surfaces of the plate as shown in Fig.1.

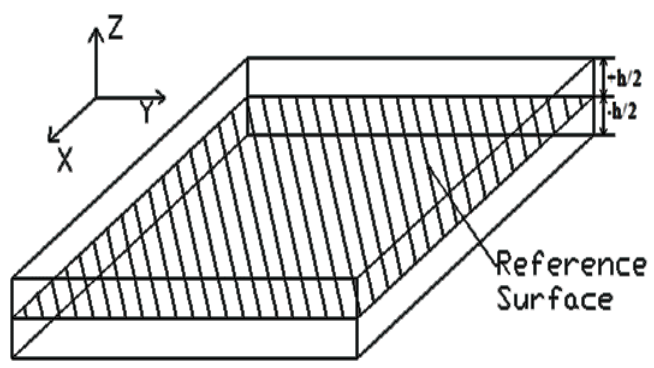

Fig.1. Coordinate system and reference surface.

\subsection{Sign convention}

The positive moment will make the upper fiber in compression and lower fiber in tension, and the negative moment will make the upper fiber in tension and lower fiber in compression. Since the curvature is proportional to the moment, therefore, the positive moment will create a positive curvature and the negative moment will create a negative curvature. As the positive moment results in a concavity on the top surface of the FGM plate, therefore, concave upward is considered to be positive and concave downward is considered to be negative.

\section{Governing equations and boundary conditions}

Kinematics of the plate theory deals with the displacement of the mid surface without considering the forces causing the displacements. The kinematic equation results in developing the relationship between strain and displacement. The plate under transverse loading, as shown in (a), bends and a slope of the midsurfaces cause displacement in the $x$ and $y$-direction as a linear function of transverse dimension (z). Let $u, v$ and, $w$ are the real displacements of the body and $\bar{u}, \bar{v}$ and $\bar{w}$ be the displacements of the mid-surface, where, $\bar{u}$ and $\bar{v}$ are in-plane displacements and $\bar{w}$ is the out of plane displacement of the mid surface as shown in (b).

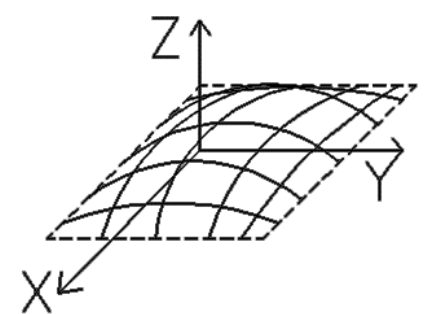

(a)

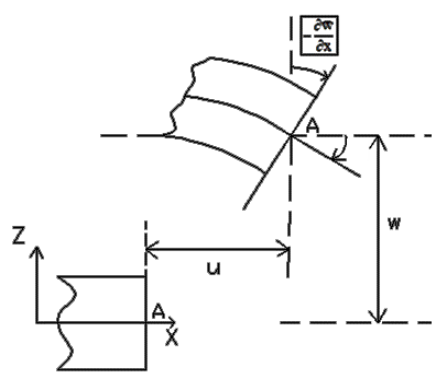

(b)

Fig.2. (a) Plate under transverse loading in the direction of $Z$ (b) Kinematics of the plate.

From the kinematics of the plate, displacement can be written as 


$$
\begin{aligned}
& u=\bar{u}-z\left(\frac{\partial \bar{w}}{\partial x}\right), \\
& v=\bar{v}-z\left(\frac{\partial \bar{w}}{\partial y}\right), \\
& w=\bar{w} .
\end{aligned}
$$

The nonlinear Lagrangian strain tensor is given as

$$
E_{i j}=\frac{1}{2}\left(\frac{\partial u_{i}}{\partial x_{j}}+\frac{\partial u_{j}}{\partial x_{i}}+\frac{\partial u_{k}}{\partial x_{i}} \frac{\partial u_{k}}{\partial x_{j}}\right)
$$

On expanding and neglecting square terms of the displacement gradient on account of small deformation but assuming the rotation of transverse normal to be moderate $\left(10^{\circ}-15^{\circ}\right)$, the following terms are not neglected, $\left(\frac{\partial w}{\partial x}\right)^{2},\left(\frac{\partial w}{\partial y}\right)^{2},\left(\frac{\partial w}{\partial x}\right)\left(\frac{\partial w}{\partial y}\right)$.

This will induce geometric nonlinearity and thus strain becomes nonlinear and denoted by epsilon $(\varepsilon)$ and substituting Eq.(3.1) in Eq.(3.2), we get

$$
\begin{aligned}
& \varepsilon_{x x}=\frac{\partial u}{\partial x}+\frac{1}{2}\left(\frac{\partial w}{\partial x}\right)^{2}=\frac{\partial \bar{u}}{\partial x}-z \frac{\partial^{2} \bar{w}}{\partial x^{2}}+\frac{1}{2}\left(\frac{\partial \bar{w}}{\partial x}\right)^{2}, \\
& \varepsilon_{y y}=\frac{\partial v}{\partial y}+\frac{1}{2}\left(\frac{\partial w}{\partial y}\right)^{2}=\frac{\partial \bar{v}}{\partial y}-z \frac{\partial^{2} \bar{w}}{\partial y^{2}}+\frac{1}{2}\left(\frac{\partial \bar{w}}{\partial y}\right)^{2}, \\
& \varepsilon_{x y}=\varepsilon_{y x}=\frac{1}{2}\left(\frac{\partial u}{\partial y}+\frac{\partial v}{\partial x}+\left(\frac{\partial w}{\partial x}\right)\left(\frac{\partial w}{\partial y}\right)\right)=\frac{1}{2}\left(\frac{\partial \bar{v}}{\partial x}+\frac{\partial \bar{u}}{\partial y}-2 z \frac{\partial^{2} \bar{w}}{\partial x \partial y}+\left(\frac{\partial \bar{w}}{\partial x}\right)\left(\frac{\partial \bar{w}}{\partial y}\right)\right),
\end{aligned}
$$

$\varepsilon_{z z}=\varepsilon_{x z}=\varepsilon_{y z}=0(\because$, there is no transverse linear and shear strain $)$

In matrices form, Eq.(3.3c) can be written as

$$
[\varepsilon]=\left[\begin{array}{c}
\varepsilon_{x x} \\
\varepsilon_{y y} \\
\varepsilon_{x y}
\end{array}\right]=\left[\begin{array}{c}
\frac{\partial \bar{u}}{\partial x}+\frac{1}{2}\left(\frac{\partial \bar{w}}{\partial x}\right)^{2} \\
\frac{\partial \bar{v}}{\partial y}+\frac{1}{2}\left(\frac{\partial \bar{w}}{\partial y}\right)^{2}\left(\frac{\partial \bar{v}}{\partial x}+\frac{\partial \bar{u}}{\partial y}+\left(\frac{\partial \bar{w}}{\partial x}\right)\left(\frac{\partial \bar{w}}{\partial y}\right)\right)
\end{array}\right]+z\left[\begin{array}{c}
-\frac{\partial^{2} \bar{w}}{\partial x^{2}} \\
-\frac{\partial^{2} \bar{w}}{\partial y^{2}} \\
-\frac{\partial^{2} \bar{w}}{\partial x \partial y}
\end{array}\right] .
$$

Thus, $\varepsilon=\varepsilon^{0}+z \varepsilon^{l}$ 
where, $\quad\left[\varepsilon^{0}\right]=\left[\begin{array}{c}\varepsilon^{0}{ }_{x x} \\ \varepsilon^{0}{ }_{y y} \\ \varepsilon^{0}{ }_{x y}\end{array}\right]=\left[\begin{array}{c}\frac{\partial \bar{u}}{\partial x}+\frac{1}{2}\left(\frac{\partial \bar{w}}{\partial x}\right)^{2} \\ \frac{\partial \bar{v}}{\partial y}+\frac{1}{2}\left(\frac{\partial \bar{w}}{\partial y}\right)^{2} \\ \frac{1}{2}\left(\frac{\partial \bar{v}}{\partial x}+\frac{\partial \bar{u}}{\partial y}+\left(\frac{\partial \bar{w}}{\partial x}\right)\left(\frac{\partial \bar{w}}{\partial y}\right)\right)\end{array}\right]=$ membrane strains (stretching),

and, $\quad\left[\varepsilon^{l}\right]=\left[\begin{array}{c}\varepsilon^{l} \\ \varepsilon_{x x}^{l} \\ y y \\ \varepsilon^{l} \\ x y\end{array}\right]=\left[\begin{array}{c}-\frac{\partial^{2} \bar{w}}{\partial x^{2}} \\ -\frac{\partial^{2} \bar{w}}{\partial y^{2}} \\ -\frac{\partial^{2} \bar{w}}{\partial x \partial y}\end{array}\right]=$ flexural or bending strains (curvature).

The linear generalized Hooke's law for an orthotropic functionally graded material is given as

$$
\sigma_{i}=C_{i j}(z) \varepsilon_{j} ; \quad i, j=1 \text { to } 6
$$

where $\sigma_{i}=$ stress vector; $\boldsymbol{C}_{i j}(\boldsymbol{z})=$ stiffness matrix; and $\varepsilon_{j}=$ engineering strain vector of the material. For an isotropic material, Hooke's law reduces to

$$
\left[\begin{array}{l}
\sigma_{x x} \\
\sigma_{y y} \\
\tau_{x y}
\end{array}\right]=\left[\begin{array}{ccc}
C_{11} & C_{12} & 0 \\
C_{12} & C_{22} & 0 \\
0 & 0 & C_{66}
\end{array}\right]\left[\begin{array}{l}
\varepsilon_{x x} \\
\varepsilon_{y y} \\
\gamma_{x y}
\end{array}\right]
$$

where, $\quad C_{11}=C_{22}=\frac{E(z)}{1-v^{2}}, \quad C_{12}=\frac{v E(z)}{1-v^{2}}=\frac{v E(z)}{1-v^{2}}, \quad C_{66}=\frac{E(z)}{2(1+v)}$.

$E(z)$ is the elastic modulus that is graded in the thickness direction and Poison's ratio $v$ is constant, $C_{i j}$ 's are reduced stiffnesses and $\sigma_{i j}$ is the Piola-Kirchhoff stress tensor.

\section{Equilibrium equation} equation

The equilibrium equation can be derived using the principle of virtual displacement given by the

$$
\delta U+\delta V=0
$$

where $\delta U=$ virtual strain energy or variation of strain energy due to internal stresses.

$\delta V=$ virtual potential energy or variation of potential energy due to external forces.

Now, the virtual strain energy $\delta U$ can be written as 


$$
\delta U=\iiint_{\sigma} \sigma: \delta \varepsilon d v=\int_{V} \sigma_{i j} \delta \varepsilon_{i j} d z d x d y
$$

where, $\sigma=$ stress tensor

$\varepsilon=$ Green Lagrange strain tensor

$:=$ operator called as double dot product .

Therefore, $\quad \delta U=\iiint\left[\sigma_{x x} \delta \varepsilon_{x x}+\sigma_{y y} \delta \varepsilon_{y y}+\sigma_{z z} \delta \varepsilon_{z z}+2\left(\sigma_{x y} \delta \varepsilon_{x y}+\sigma_{y z} \delta \varepsilon_{y z}+\sigma_{z x} \delta \varepsilon_{z x}\right)\right] d v$.

In the stiff plate problem,

$$
\delta \varepsilon_{z z}=\delta \varepsilon_{y z}=\delta \varepsilon_{z x}=0
$$

Thus, substituting the above in Eq.(4.3), we get

$$
\delta U=\iiint\left[\sigma_{x x} \delta \varepsilon_{x x}+\sigma_{y y} \delta \varepsilon_{y y}+2 \sigma_{x y} \delta \varepsilon_{x y}\right] d v .
$$

Taking a variation of it, we get

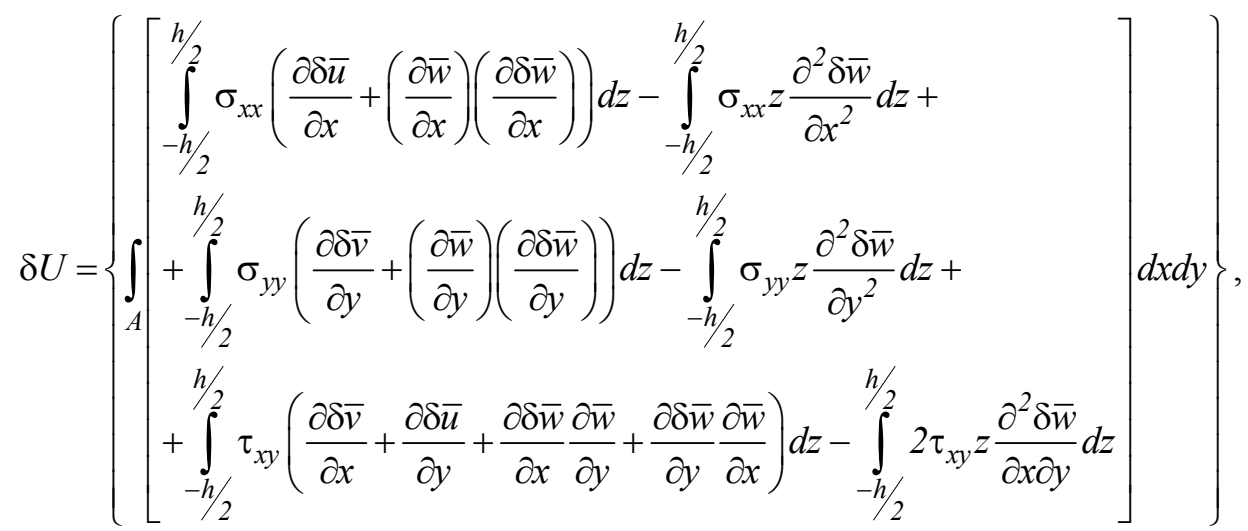

$$
\begin{aligned}
& \delta U=\int_{A}\left[\left(N_{x x} \delta \varepsilon_{x x}^{0}+N_{y y} \delta \varepsilon_{y y}^{0}+N_{x y} \delta \varepsilon_{x y}^{0}\right)-\left(M_{x x} \delta \varepsilon_{x x}^{1}+M_{y y} \delta \varepsilon_{y y}^{1}+2 M_{x y} \delta \varepsilon_{x y}^{l}\right)\right] d A
\end{aligned}
$$

The virtual work done by the external force is given as

$$
\delta V=-\iint q_{z} \delta \bar{w} d x d y
$$

where, $q_{z}=$ distributed transverse load applied on the top of the surface

$\delta \bar{w}=$ applied virtual displacement

Now, substituting Eqs (4.4) and (4.5) into Eq.(4.1), we get

$$
\begin{aligned}
& \delta W=\int_{A}\left[\left(N_{x x} \delta \varepsilon_{x x}^{0}+N_{y y} \delta \varepsilon_{y y}^{0}+N_{x y} \delta \varepsilon_{x y}^{0}\right)+\right. \\
& \left.-\left(M_{x x} \delta \varepsilon_{x x}^{1}+M_{y y} \delta \varepsilon_{y y}^{1}+2 M_{x y} \delta \varepsilon_{x y}^{1}\right)-q_{z} \delta w\right] d A .
\end{aligned}
$$


Now solving the above equation term by term and on rearranging, we get

$$
\begin{aligned}
& \delta W=\int_{A}\left\{\left(-\frac{\partial N_{x x}}{\partial x}-\frac{\partial N_{x y}}{\partial y}\right) \delta \bar{u}+\left(-\frac{\partial N_{y y}}{\partial y}-\frac{\partial N_{x y}}{\partial x}\right) \delta \bar{v}+\right. \\
& \left.+\left(\begin{array}{l}
-\frac{\partial}{\partial x}\left(N_{x x} \frac{\partial \bar{w}}{\partial x}+N_{x y} \frac{\partial \bar{w}}{\partial y}\right)-\frac{\partial}{\partial y}\left(N_{y y} \frac{\partial \bar{w}}{\partial y}+N_{x y} \frac{\partial \bar{w}}{\partial x}\right)+ \\
-\left(\frac{\partial^{2} M_{x x}}{\partial x^{2}}+\frac{\partial^{2} M_{y y}}{\partial y^{2}}+2 \frac{\partial^{2} M_{x y}}{\partial x \partial y}-q_{z}\right.
\end{array}\right) \delta \bar{w}\right\} d A+ \\
& +\oint_{\Gamma}\left\{\begin{array}{l}
\left(N_{x x} n_{x}+N_{x y} n_{y}\right) \delta \bar{u}+\left(N_{y y} n_{y}+N_{x y} n_{x}\right) \delta \bar{u}+\left[\begin{array}{l}
\left(N_{x x} n_{x}+N_{x y} n_{y}\right) \frac{\partial \bar{w}}{\partial x}+\left(N_{y y} n_{y}+N_{x y} n_{x}\right) \frac{\partial \bar{w}}{\partial y} \\
+\left(\frac{\partial M_{x x}}{\partial x}+\frac{\partial M_{y x}}{\partial y}\right) n_{x}+\left(\frac{\partial M_{y y}}{\partial y}+\frac{\partial M_{x y}}{\partial x}\right)
\end{array}\right) \delta \bar{w} \\
\left(M_{x x} n_{x}+M_{y x} n_{y}\right) \frac{\partial \delta \bar{w}}{\partial x}-\left(M_{y y} n_{y}+M_{x y} n_{x}\right) \frac{\partial \delta \bar{w}}{\partial y}
\end{array}\right\} d s .
\end{aligned}
$$

The Euler-Lagrange equation can be obtained by the fundamental lemma of variational calculus as

$$
\begin{aligned}
& \delta \bar{u}: \frac{\partial N_{x x}}{\partial x}+\frac{\partial N_{x y}}{\partial y}=0, \\
& \delta \bar{v}: \frac{\partial N_{y y}}{\partial y}+\frac{\partial N_{x y}}{\partial x}=0, \\
& \delta \bar{w}:\left(\frac{\partial^{2} M_{x x}}{\partial x^{2}}+\frac{\partial^{2} M_{y y}}{\partial y^{2}}+2 \frac{\partial^{2} M_{x y}}{\partial x \partial y}+q_{z}\right)+\frac{\partial}{\partial x}\left(N_{x x} \frac{\partial \bar{w}}{\partial x}+N_{x y} \frac{\partial \bar{w}}{\partial y}\right)+ \\
& +\frac{\partial}{\partial y}\left(N_{y y} \frac{\partial \bar{w}}{\partial y}+N_{x y} \frac{\partial \bar{w}}{\partial x}\right)=0 .
\end{aligned}
$$

The boundary conditions are given by equating the term equal to zero of domain $\Gamma$. We get

$$
\begin{array}{lll}
\delta \bar{u}=0 & \text { or } & N_{x x} n_{x}+N_{x y} n_{y}=0, \\
\delta \bar{v}=0 \quad \text { or } & N_{y y} n_{y}+N_{x y} n_{x}=0 \\
\delta \bar{w}=0 \quad \text { or } & {\left[\begin{array}{l}
\left.\left(N_{x x} n_{x}+N_{x y} n_{y}\right) \frac{\partial \bar{w}}{\partial x}+\left(N_{y y} n_{y}+N_{x y} n_{x}\right) \frac{\partial \bar{w}}{\partial y}+\right] \\
+\left(\frac{\partial M_{x x}}{\partial x}+\frac{\partial M_{y x}}{\partial y}\right) n_{x}+\left(\frac{\partial M_{y y}}{\partial y}+\frac{\partial M_{x y}}{\partial x}\right) n_{y}
\end{array}\right]=0,}
\end{array}
$$




$$
\begin{array}{lll}
\delta\left(\frac{\partial \bar{w}}{\partial x}\right)=0 & \text { or } & -\left(M_{x x} n_{x}+M_{y x} n_{y}\right)=0, \\
\delta\left(\frac{\partial \bar{w}}{\partial y}\right)=0 & \text { or } & -\left(M_{y y} n_{y}+M_{x y} n_{x}\right)=0 .
\end{array}
$$

\subsection{Relationship between stress resultants and middle surface displacements}

The plate theory reduces the 3-D continuum problem to a 2-D Lamina problem by applying certain assumptions, thus, the plate is treated as a plane and therefore all the quantities depend on $x$ and $y$ alone. This is done by integrating the quantities in the $z$ - direction i.e., through the plate thickness and thus the threedimensional problem reduces to two-dimensional. Therefore, the three stress components on the cross section are replaced by a set of resultant forces and moments and are known as stress resultants. Mathematically, a stress resultant is defined as the integral of stress over the thickness of the plate yielding the following set of stress resultants

$$
\left\{\begin{array}{l}
N_{x x} \\
N_{y y} \\
N_{x y}
\end{array}\right\}=\int_{-h / 2}^{h / 2}\left\{\begin{array}{l}
\sigma_{x x} \\
\sigma_{y y} \\
\tau_{x y}
\end{array}\right\} d z \quad \text { and } \quad\left\{\begin{array}{l}
M_{x x} \\
M_{y y} \\
M_{x y}
\end{array}\right\}=\int_{-h / 2}^{h / 2} z\left\{\begin{array}{l}
\sigma_{x x} \\
\sigma_{y y} \\
\tau_{x y}
\end{array}\right\} d z
$$

Now, stress resultants and moment resultants given in terms of stiffness are

$$
\begin{aligned}
& \left\{\begin{array}{l}
N_{x x} \\
N_{y y} \\
N_{x y}
\end{array}\right\}=\left[\begin{array}{ccc}
A_{11} & A_{12} & 0 \\
A_{12} & A_{22} & 0 \\
0 & 0 & A_{66}
\end{array}\right]\left\{\begin{array}{l}
\varepsilon^{0}{ }_{x x} \\
\varepsilon^{0}{ }_{y y} \\
\gamma^{0}{ }_{x y}
\end{array}\right\}+\left[\begin{array}{ccc}
B_{11} & B_{12} & 0 \\
B_{12} & B_{22} & 0 \\
0 & 0 & B_{66}
\end{array}\right]\left\{\begin{array}{l}
\varepsilon^{1}{ }_{x x} \\
\varepsilon^{1}{ }_{y y} \\
\gamma_{x y}^{1}
\end{array}\right\}, \\
& \left\{\begin{array}{l}
M_{x x} \\
M_{y y} \\
M_{x y}
\end{array}\right\}=\left[\begin{array}{ccc}
B_{11} & B_{12} & 0 \\
B_{12} & B_{22} & 0 \\
0 & 0 & B_{66}
\end{array}\right]\left\{\begin{array}{l}
\varepsilon_{x x}^{0} \\
\varepsilon^{0} \\
\gamma_{x y}^{0}
\end{array}\right\}+\left[\begin{array}{ccc}
D_{11} & D_{12} & 0 \\
D_{12} & D_{22} & 0 \\
0 & 0 & D_{66}
\end{array}\right]\left\{\begin{array}{c}
\varepsilon_{x x}{ }^{1} \\
\varepsilon_{y y}{ }^{1} \\
\gamma_{x y}{ }^{1}
\end{array}\right\},
\end{aligned}
$$

and in a compact form

$$
\left\{\begin{array}{l}
N \\
M
\end{array}\right\}=\left[\begin{array}{ll}
A & B \\
B & D
\end{array}\right]\left\{\begin{array}{l}
\varepsilon^{0} \\
\varepsilon^{l}
\end{array}\right\}
$$


where, $A_{i j}=\int_{-h / 2}^{h / 2} C_{i j}(z) d z=$ extensional stiffnesses , $B_{i j}=\int_{-h / 2}^{h / 2} z C_{i j}(z) d z=$ bending-extensional stiffnesses,

$D_{i j}=\int_{-h / 2}^{h / 2} z^{2} C_{i j}(z) d z=$ bending stiffnesses .

The expressions for the curvatures and slopes defined in Eq.(3.4) are now substituted in Eqs (4.9) and (4.10) and finally substituted in Eqs (4.8a) - (4.8c) to obtain the equilibrium equations in terms of displacements. The equilibrium equation in terms of displacement can be written as

$$
\begin{aligned}
& \left\{A_{11}\left[\frac{\partial^{2} \bar{u}}{\partial x^{2}}+\left(\frac{\partial \bar{w}}{\partial x}\right)\left(\frac{\partial^{2} \bar{w}}{\partial x^{2}}\right)\right]+A_{12}\left[\frac{\partial^{2} \bar{v}}{\partial x \partial y}+\left(\frac{\partial \bar{w}}{\partial y}\right)\left(\frac{\partial^{2} \bar{w}}{\partial x \partial y}\right)\right]+A_{66}\left(\frac{\partial^{2} \bar{v}}{\partial x \partial y}+\frac{\partial^{2} \bar{u}}{\partial y^{2}}+\right.\right. \\
& \left.\left.+\left(\frac{\partial^{2} \bar{w}}{\partial y^{2}}\right)\left(\frac{\partial \bar{w}}{\partial x}\right)+\left(\frac{\partial^{2} \bar{w}}{\partial x \partial y}\right)\left(\frac{\partial \bar{w}}{\partial y}\right)\right)-B_{11}\left(\frac{\partial^{3} \bar{w}}{\partial x^{3}}\right)-\left(B_{12}+2 B_{66}\right)\left(\frac{\partial^{3} \bar{w}}{\partial x \partial y^{2}}\right)\right\}=0 \\
& \left\{A_{12}\left[\frac{\partial^{2} \bar{u}}{\partial x \partial y}+\left(\frac{\partial \bar{w}}{\partial x}\right)\left(\frac{\partial^{2} \bar{w}}{\partial x \partial y}\right)\right]+A_{22}\left[\frac{\partial^{2} \bar{v}}{\partial y^{2}}+\left(\frac{\partial \bar{w}}{\partial y}\right)\left(\frac{\partial^{2} \bar{w}}{\partial y^{2}}\right)\right]+A_{66}\left[\frac{\partial^{2} \bar{v}}{\partial x^{2}}+\frac{\partial^{2} \bar{u}}{\partial x \partial y}+\right.\right. \\
& \left.\left.+\left(\frac{\partial^{2} \bar{w}}{\partial x^{2}}\right)\left(\frac{\partial \bar{w}}{\partial y}\right)+\left(\frac{\partial^{2} \bar{w}}{\partial x \partial y}\right)\left(\frac{\partial \bar{w}}{\partial x}\right)\right]-B_{22}\left(\frac{\partial^{3} \bar{w}}{\partial y^{3}}\right)-\left(B_{12}+2 B_{66}\right)\left(\frac{\partial^{3} \bar{w}}{\partial x^{2} \partial y}\right)\right\}=0 \\
& \left\{B_{11}\left[\frac{\partial^{3} \bar{u}}{\partial x^{3}}+\left(\frac{\partial^{2} \bar{w}}{\partial x^{2}}\right)^{2}+\left(\frac{\partial \bar{w}}{\partial x}\right)\left(\frac{\partial^{3} \bar{w}}{\partial x^{3}}\right)\right]+B_{22}\left[\frac{\partial^{3} \bar{v}}{\partial y^{3}}+\left(\frac{\partial^{2} \bar{w}}{\partial y^{2}}\right)^{2}+\left(\frac{\partial \bar{w}}{\partial y}\right)\left(\frac{\partial^{3} \bar{w}}{\partial y^{3}}\right)\right]+\right. \\
& +B_{12}\left(\frac{\partial^{2} \bar{w}}{\partial x \partial y}\right)^{2}+2 B_{66}\left(\frac{\partial^{2} \bar{w}}{\partial y^{2}}\right)\left(\frac{\partial^{2} \bar{w}}{\partial x^{2}}\right)+\left(B_{12}+2 B_{66}\right)\left[\frac{\partial^{3} \bar{u}}{\partial x \partial y^{2}}+\left(\frac{\partial^{2} \bar{w}}{\partial x \partial y}\right)^{2}+\right. \\
& \left.+\left(\frac{\partial \bar{w}}{\partial x}\right)\left(\frac{\partial^{3} \bar{w}}{\partial x \partial y^{2}}\right)+\frac{\partial^{3} \bar{v}}{\partial x^{2} \partial y}+\left(\frac{\partial \bar{w}}{\partial y}\right)\left(\frac{\partial^{3} \bar{w}}{\partial x^{2} \partial y}\right)\right]-D_{11} \frac{\partial^{4} \bar{w}}{\partial x^{4}}-2\left(D_{12}+2 D_{66}\right)\left(\frac{\partial^{4} \bar{w}}{\partial x^{2} \partial y^{2}}\right)+ \\
& \left.-D_{22} \frac{\partial^{4} \bar{w}}{\partial y^{4}}+\frac{\partial}{\partial x}\left(N_{x x} \frac{\partial \bar{w}}{\partial x}+N_{x y} \frac{\partial \bar{w}}{\partial y}\right)+\frac{\partial}{\partial y}\left(N_{y y} \frac{\partial \bar{w}}{\partial y}+N_{x y} \frac{\partial \bar{w}}{\partial x}\right)\right\}=-q_{z} \cdot
\end{aligned}
$$

\section{Analytical solution}

Navier's type solution is used to obtain the deflections and stresses for the functionally graded plate. The displacement fields that satisfy the boundary conditions and the governing equilibrium equations can be written as follows

$$
\bar{u}=\sum_{m=1}^{\infty} \sum_{n=1}^{\infty} U_{m n} \sin \left(\frac{m \pi x}{a}\right) \sin \left(\frac{n \pi y}{b}\right)
$$




$$
\begin{aligned}
& \bar{v}=\sum_{m=1}^{\infty} \sum_{n=1}^{\infty} V_{m n} \sin \left(\frac{m \pi x}{a}\right) \sin \left(\frac{n \pi y}{b}\right), \\
& \bar{w}=\sum_{m=1}^{\infty} \sum_{n=1}^{\infty} W_{m n} \sin \left(\frac{m \pi x}{a}\right) \sin \left(\frac{n \pi y}{b}\right) .
\end{aligned}
$$

The transverse load $q_{z}(x, y)$ using the double Fourier series is written as

$$
q_{z}(x, y)=\sum_{m=1}^{\infty} \sum_{n=1}^{\infty} Q_{m n} \sin \left(\frac{m \pi x}{a}\right) \sin \left(\frac{n \pi y}{b}\right)
$$

where

$$
Q_{m n}=\frac{4}{a b} \int_{0}^{a} \int_{0}^{b} q_{z}(x, y) \sin \left(\frac{m \pi x}{a}\right) \sin \left(\frac{n \pi y}{b}\right) d x d y .
$$

Let us assume the solution for uniform loading of $q_{0}$, that is, $q_{z}(x, y)=q_{0}$.

Therefore, $\quad Q_{m n}=\frac{16 q_{0}}{m n \pi^{2}}$.

Thus, the transverse load $q_{z}(x, y)$ is defined as

$$
q_{z}(x, y)=\frac{16 q_{0}}{\pi^{2}} \sum_{m=1}^{\infty} \sum_{n=1}^{\infty} \frac{\sin \left(\frac{m \pi x}{a}\right) \sin \left(\frac{n \pi y}{b}\right)}{m n} .
$$

The governing equation in a matrix form can be written as

$$
\left(\begin{array}{ccccccc}
s_{11} & s_{12} & s_{13} & 0 & 0 & s_{16} & 0 \\
s_{21} & s_{22} & s_{23} & 0 & 0 & s_{26} & 0 \\
s_{31} & s_{32} & s_{33} & s_{34} & s_{35} & s_{36} & s_{37}
\end{array}\right)\left(\begin{array}{c}
U \\
V \\
W \\
U W \\
V W \\
W^{2} \\
W^{3}
\end{array}\right)=\left(\begin{array}{l}
0 \\
0 \\
S
\end{array}\right)
$$

where

$$
\begin{aligned}
& s_{11}=-\sin \alpha x \sin \beta y\left(\alpha^{2} A_{11}+\beta^{2} A_{66}\right), \quad s_{12}=\alpha \beta \cos \alpha x \cos \beta y\left(A_{12}+A_{66}\right), \\
& s_{13}=\alpha \cos \alpha x \sin \beta y\left(\alpha^{2} B_{11}+\beta^{2}\left(B_{12}+2 B_{66}\right)\right), \\
& s_{16}=\frac{1}{4} \alpha \sin 2 \alpha x\left(\cos (2 \beta y)\left(\alpha^{2} A_{11}+\beta^{2}\left(A_{12}+2 A_{66}\right)\right)+\beta^{2} A_{12}-\alpha^{2} A_{11}\right), \\
& s_{21}=\alpha \beta \cos \alpha x \cos \beta y\left(A_{12}+A_{66}\right), \quad s_{22}=-\sin \alpha x \sin \beta y\left(\alpha^{2} A_{66}+\beta^{2} A_{22}\right),
\end{aligned}
$$




$$
\begin{aligned}
& s_{23}=\beta \sin \alpha x \cos \beta y\left(\alpha^{2}\left(B_{12}+2 B_{66}\right)+\beta^{2} B_{22}\right), \\
& s_{26}=\frac{1}{4} \beta \sin 2 \beta y\left(\cos 2 \alpha x\left(\alpha^{2}\left(A_{12}+2 A_{66}\right)+A_{22} \beta^{2}\right)+\alpha^{2} A_{12}-A_{22} \beta^{2}\right), \\
& s_{31}=\alpha \cos \alpha x \sin \beta y\left(\alpha^{2} B_{11}+\beta^{2}\left(B_{12}+2 B_{66}\right)\right), \\
& s_{32}=-\beta \sin \alpha x \cos \beta y\left(\alpha^{2}\left(B_{12}+2 B_{66}\right)+\beta^{2} B_{22}\right), \\
& s_{33}=-\sin \alpha x \sin \beta y\left(2 \alpha^{2} \beta^{2}\left(D_{12}+2 D_{66}\right)+\left(\beta^{4} D_{22}+\alpha^{4} D_{11}\right)\right), \\
& s_{34}=\frac{1}{2} \alpha \sin 2 \alpha x\left(\cos 2 \beta y\left(\alpha^{2} A_{11}+\beta^{2}\left(A_{12}+2 A_{66}\right)\right)+\beta^{2} A_{66}-\alpha^{2} A_{11}\right), \\
& s_{35}=\frac{1}{2} \beta \sin 2 \beta y\left(\cos 2 \alpha x\left(\alpha^{2}\left(A_{12}+2 A_{66}\right)+A_{22} \beta^{2}\right)+\alpha^{2} A_{66}-A_{22} \beta^{2}\right), \\
& s_{36}=2 \alpha^{2} W^{2}\left(\sin { }^{2} \alpha x \sin ^{2} \beta y\left(\alpha^{2} B_{66}-\beta^{2} B_{12}\right)+\beta^{2} \cos ^{2} \alpha x \cos ^{2} \beta y\left(B_{12}-B_{66}\right)\right), \\
& s_{37}=\sin (\alpha x) \sin (\beta y)\left[\left(\alpha ^ { 2 } \operatorname { c o s } ^ { 2 } \alpha x \left(-0.75 \alpha^{2} \mathrm{~A}_{11}+\cos _{2} \beta y\left(0.75 \alpha^{2} A_{11}+\beta^{2}\left(1.25 A_{12}+2.5 A_{66}\right)\right)+\right.\right.\right. \\
& \left.\left.\left.+\beta^{2}\left(0.75 A_{12}+1.5 A_{66}\right)\right)-\beta^{2} \sin ^{2} \alpha x \cos ^{2} \beta y\left(0.5 \alpha^{2} A_{12}+1.5 A_{22} \beta^{2}+\alpha^{2} A_{66}\right)\right)\right], \\
& S_{=}-\frac{16 q_{0} \sin (\alpha x) \sin (\beta y)}{\pi^{2} m n} \quad \alpha=\frac{m \pi}{a}, \quad \beta \pi
\end{aligned}
$$

\section{Numerical results and discussions}

A functionally graded plate with a different span-to-thickness ratio $(a / h)$ is investigated to analyze the simply supported square plates $(a=b)$ subjected to uniform transverse load $\left(q_{\mathrm{z}}\right)$ on the top surface $(z=h / 2), q_{0}=-1 \times 10^{5} \mathrm{~N} / \mathrm{m}^{2}$. Six different span-to-thickness ratios considered are 4, 10, 50, 70, 80, and 100 for analyzing the maximum central deflections.

The plate properties are graded from Aluminium (bottom) to Alumina (top) using rules of mixture. Keeping the temperature and Poisson ratio constant, the Young modulus is graded in the thickness direction using the following functional relationship

$$
\mathrm{E}(\mathrm{z})=\mathrm{E}_{\mathrm{m}}+\left(\mathrm{E}_{\mathrm{c}}-\mathrm{E}_{\mathrm{m}}\right)\left(\frac{1}{2}+\frac{z}{h}\right)^{f}
$$

where $\mathrm{E}_{\mathrm{m}}=70 \mathrm{GPa}$ and $\mathrm{E}_{\mathrm{c}}=380 \mathrm{GPa}$ are the Young modulus for the metal (Aluminium) and ceramic (Alumina) respectively; $f$ is the volume fraction exponent; $h$ is the thickness of the plate. The Poisson ratio (v) for both metal and ceramic is 0.3 . 


\subsection{Analysis of deflection}

In deflection analysis, six different volume fraction exponents are considered, viz., $0.5,2,4,6,8$, and 10. On applying Navier's solution, the solution so obtained is complex and plotted on a complex plane as shown in Fig.3 and the absolute solution is shown in Fig.4. The following observations have been made for the functionally graded and pure metal and ceramic plate for the complex solution of deflection:

1) The solution of deflections in the case of pure metal and ceramic has only approximately real part with the imaginary part approaching zero, but for functionally graded part the solution is complex.

2) Although the magnitude of the imaginary part of deflection is very small, still it shows the real behavior of the plate deflection in the context of the volume fraction exponent.

3 ) With decreasing the thickness for the case of a stiff plate $(a / h=10$ to 50$)$, the effect of gradation of mechanical properties reduces and there will be a little difference between deflections for different volume fraction exponents, so the first peak for $a / h=4$ takes place at volume fraction 2 and it goes on increasing to 6 and 8 in the case of $a / h=10$ and 50 , respectively.

4) As thickness goes on decreasing, in the case of membranes $(a / h=80$ to 100$)$, the behavior reverses and deflection of the plate is due to the presence of metal and little effect of the functionally graded material according to the NLCPT.
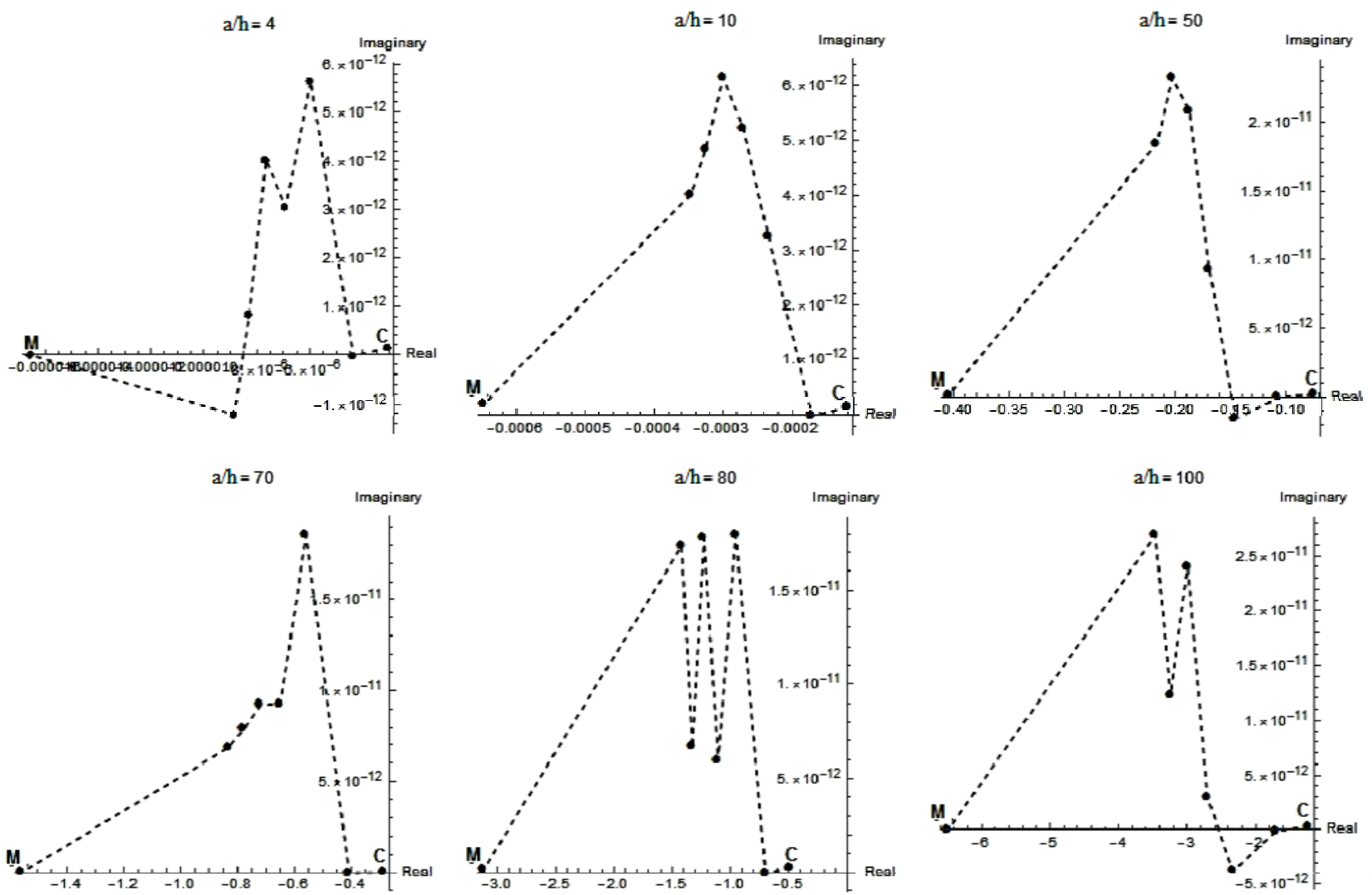

Fig.3. Complex solution of deflection/thickness of the functionally graded plate for various volume fraction exponents $(\mathrm{C}, 0.5,2,4,6,8,10, \mathrm{M})$ and span-to-thickness ratios $(4,10,50,70,80,100)(\mathrm{C}$ and $\mathrm{M}$ stands for ceramic and metal, respectively).

The following observations have been made for the functionally graded and pure metal and ceramic plate for the absolute solution of deflection based on Fig.4:

1) For a thick $(a / h<10)$ and stiff $(a / h=10-50)$ plate, there is a gradual increase in the deflection of the plate for the constant volume fraction exponent (excluding metal). 
2) For a flexible plate $(a / h=50-80)$, there is a constant increase in the deflection of the plate for the constant volume fraction exponent (excluding metal).

3) For membranes $(a / h=80-100)$, there is a sudden increase in the deflection of the plate for the constant volume fraction exponent (excluding metal).

4) In the case of metal, there is a gradual increase in the deflection for a thick, stiff and a flexible plate but a sudden increase in deflection takes place for membranes.

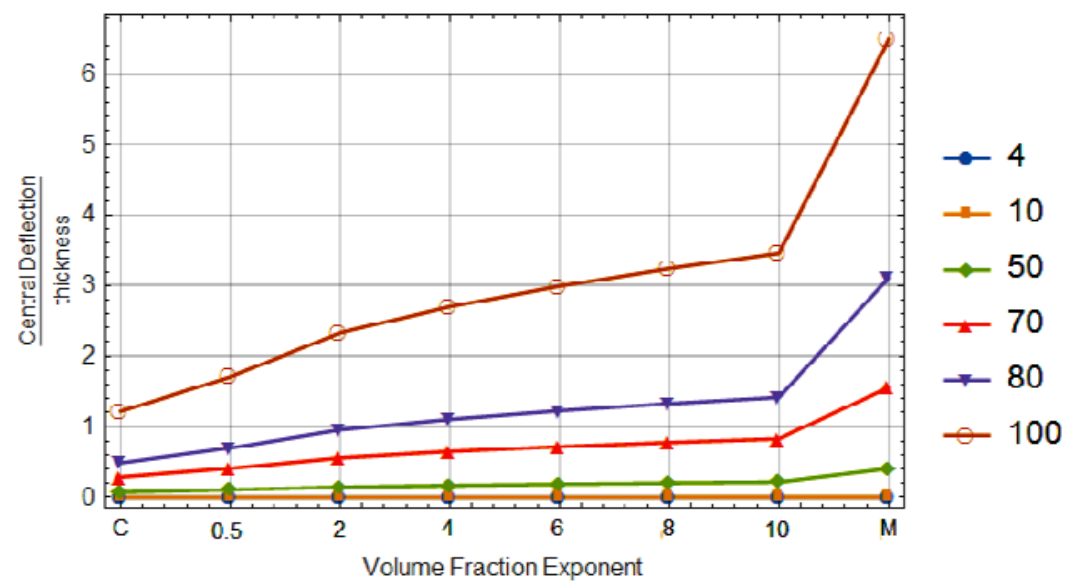

Fig.4. Absolute solution of deflection/thickness of the functionally graded plate for various volume fraction exponents and span-to-thickness ratios $(\mathrm{C}$ and $\mathrm{M}$ stands for ceramic and metal, respectively).

\subsection{Analysis of stresses}

Two different aspects are analyzed in the stress analysis:

(i) the effect of variation of the volume fraction exponent on stress,

(ii) variation of stress along the thickness.

\subsubsection{Effect of variation of volume fraction exponent $(f)$ on stress}

Ten different volume fraction exponents are considered, 1-10. On applying Navier's solution, the solution obtained is complex, so the analysis for stresses is made on the basis of a real and absolute solution.

The following observations have been made for the functionally graded and pure metal and ceramic plate stresses:

1) The stresses in the functionally graded material lie in between the stresses in metal and ceramic due to a reduction in stiffness property of a material which lies in between metal and ceramic.

2) For metal, the actual value of stresses is always greater than for the real part of the solution as shown in Fig.5 - Fig.8.

3) The stresses on top of the plate are always greater than those of the bottom of the fibers and also of an opposite nature for the functionally graded material as shown in Fig.5 - Fig.8.

4) For ceramics and metals, the normal stresses at $z= \pm h / 2$ of the plate are approximately equal as shown in Fig.5 - Fig.6.

5) A negligible amount of shear stresses is induced for a thick $(a / h<10)$, stiff $(a / h=10-50)$ and flexible plate $(a / h=50-80)$ but a considerable amount of shear stresses is induced in the case of membranes $(a / h$ $=80-100)$ as shown in Fig.7 - Fig.8. 


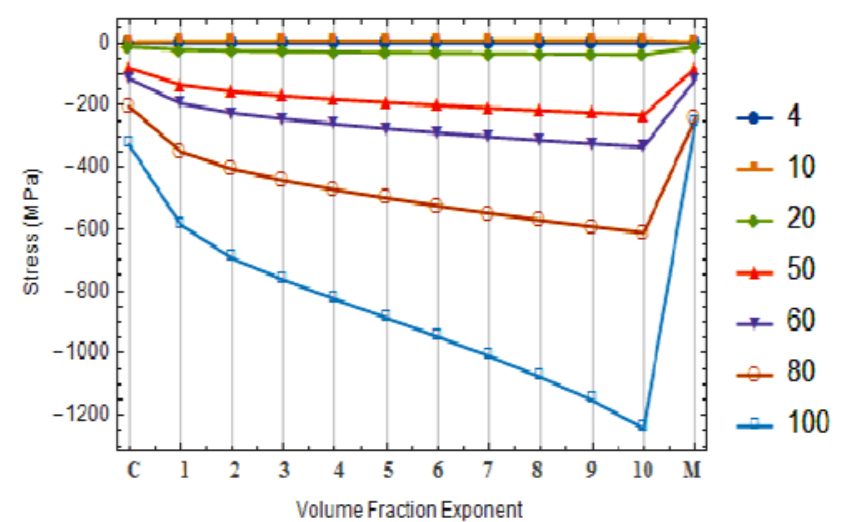

(a)

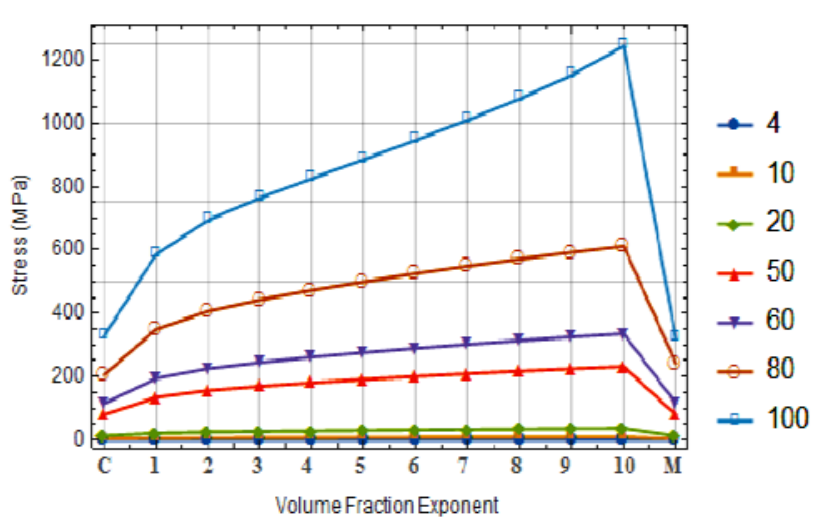

(b)

Fig.5. (a) Real and (b) absolute solution for normal stresses $\left(\sigma_{X X}, \sigma_{Y Y}\right)$ in the $X$ or $Y$-direction at $z=+h / 2$ for various volume fraction exponents and span-to-thickness ratios $(\mathrm{C}$ and $\mathrm{M}$ stands for ceramic and metal respectively).

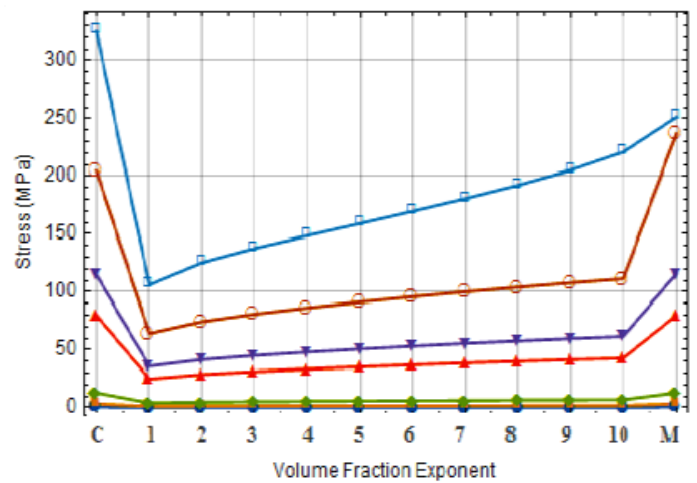

(a)

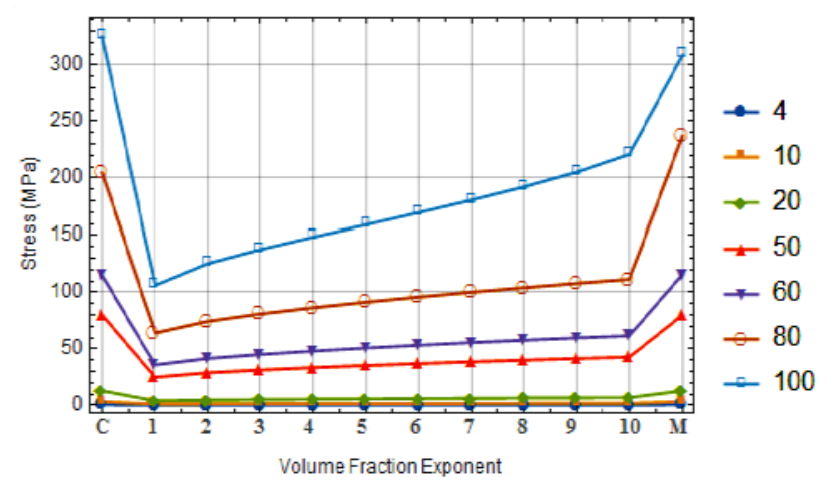

(b)

Fig.6. (a) Real and (b) absolute solution for normal stresses $\left(\sigma_{X X}, \sigma_{Y Y}\right)$ in the $X$ or $Y$-direction at $z=-h / 2$ for various volume fraction exponents and span-to-thickness ratios $(\mathrm{C}$ and $\mathrm{M}$ stands for ceramic and metal, respectively).

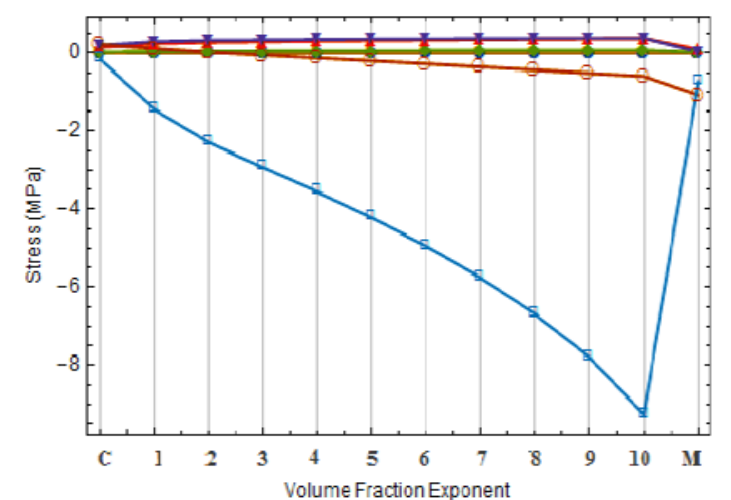

(a)

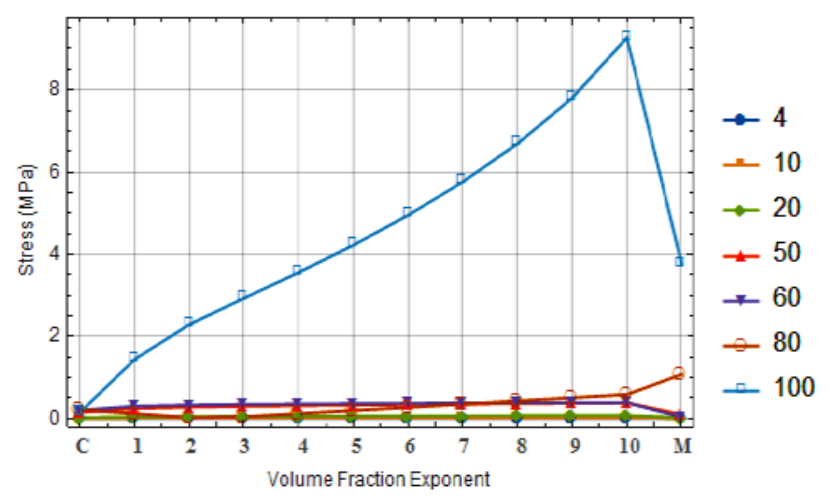

(b)

Fig.7. (a) Real and (b) absolute solution for shear stresses $\left(\sigma_{X Y}\right)$ at $z=+h / 2$ for various volume fraction exponents and span-to-thickness ratios ( $\mathrm{C}$ and $\mathrm{M}$ stands for ceramic and metal, respectively). 


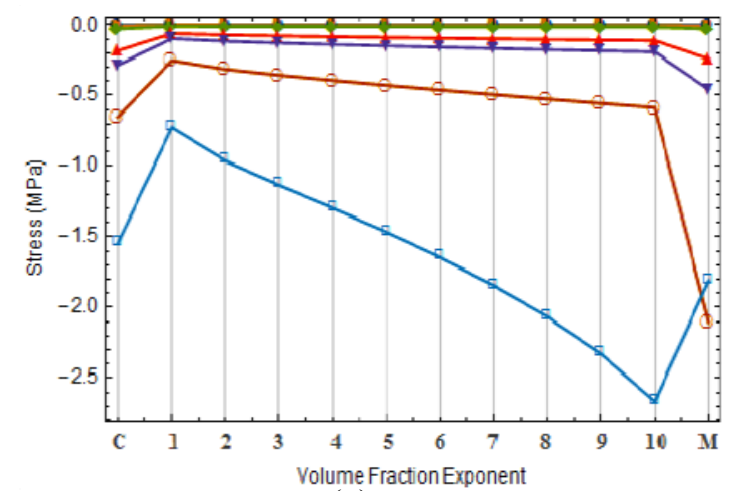

(a)

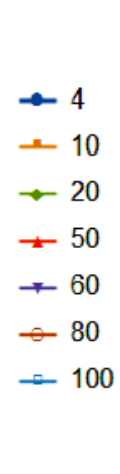

$-4$

$-20$

$+50$

$-100$

\section{0}

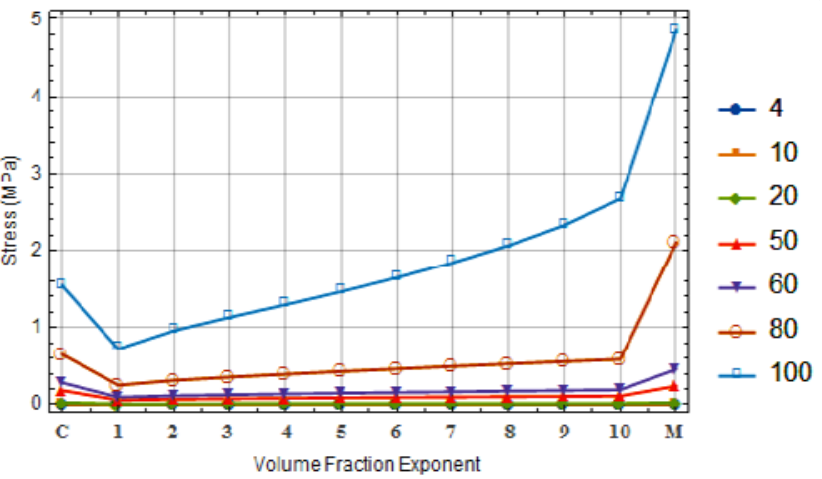

(b)

Fig.8. (a) Real and (b) absolute solution for the shear stresses $\left(\sigma_{X Y}\right)$ at $z=-h / 2$ for various volume fraction exponents and span-to-thickness ratios ( $\mathrm{C}$ and $\mathrm{M}$ stands for ceramic and metal, respectively).

\subsubsection{Variation of stress along the thickness}

Three different volume fraction exponents $(1,4$, and 10) with $a / h=4$ are considered. On applying Navier's solution, the solution so obtained was complex and plotted on a complex plane as shown in Figs 9 and 12 and the real part of the solution is shown in s Figs 13 and 16. The stresses in the transverse direction are neglected according to the classical plate theory, but transverse stresses can be calculated using 3D elasticity equation. So, along with normal and shear stresses, a variation of transverse stresses along the thickness is also considered.

The following observations have been made for the functionally graded plate for the complex solution of stresses:

1) The variation of stresses, excluding transverse stresses, across the thickness are uniformly distributed when the volume fraction exponent exhibits a linear behavior but as its value increases from 1 to 10 , the stresses are distributed in a non-uniform way and stresses are more concentrated towards the bottom surface of the plate but dispersed on the top surface of the plate as shown in Fig.9 and Fig.11.

2) The transverse normal stress does not depend on the volume fraction exponent and, therefore, does not exhibit any transformation on the changing volume fraction exponent according to NLCPT as shown in Fig.10.

3) The transverse shear stress shows a reverse behavior compared to linear stresses in a way such that it is more concentrated at the top of the plate and dispersed towards the bottom of the plate, as the volume fraction exponent increases as shown in Fig.12.

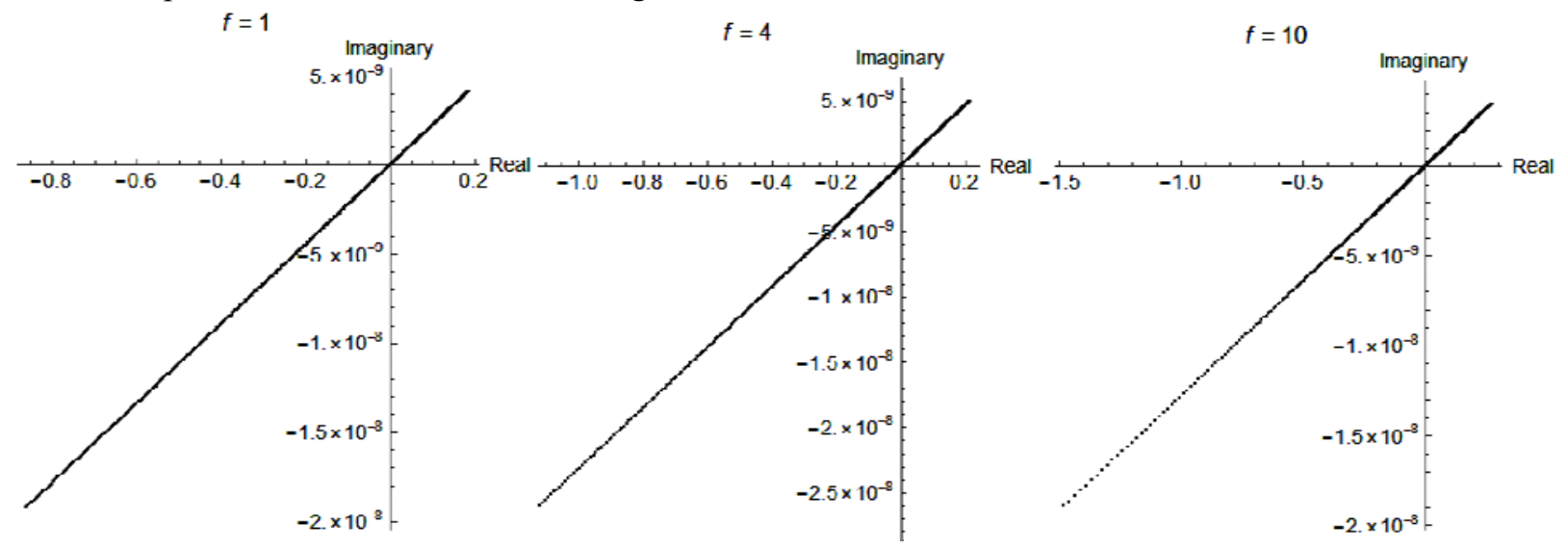

Fig.9. Complex solution for normal stresses $\left(\sigma_{X X}, \sigma_{Y Y}\right)$ in the $X$ or $Y$-direction across the thickness for the volume fraction exponent (f) and $a / h=4$. 


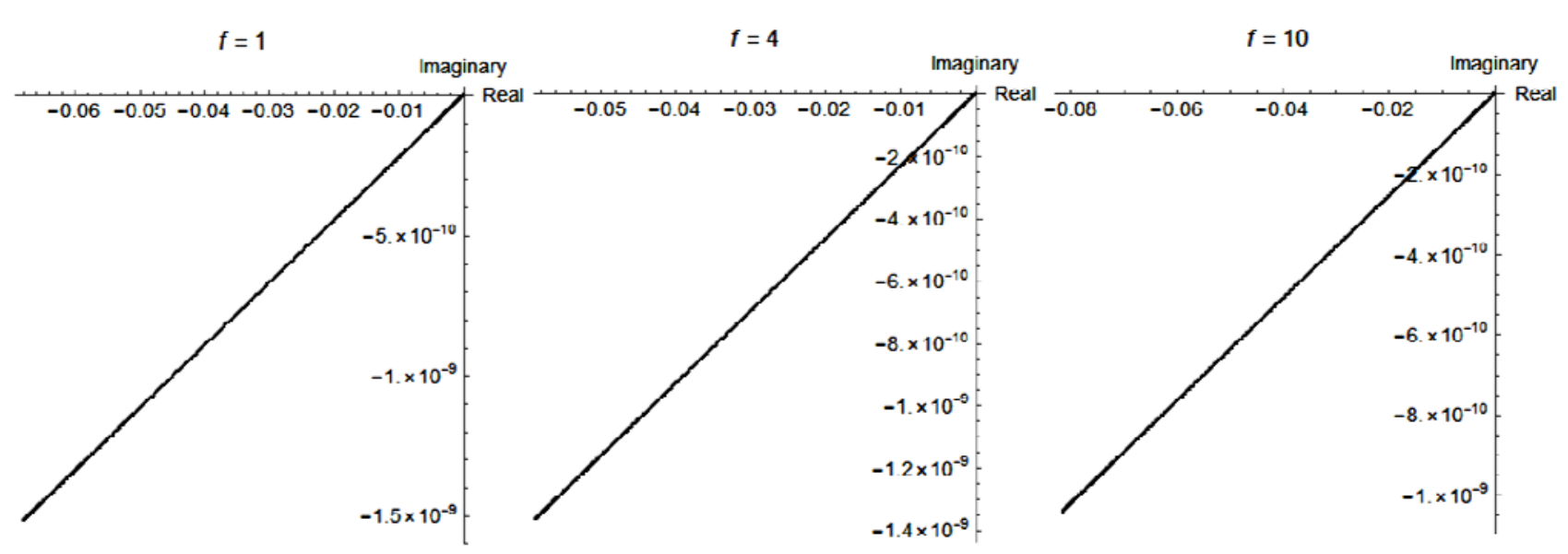

Fig.10. Complex solution for transverse normal stresses $\left(\sigma_{Z Z}\right)$ in the $Z$-direction across the thickness for the volume fraction exponent (f) and $a / h=4$.
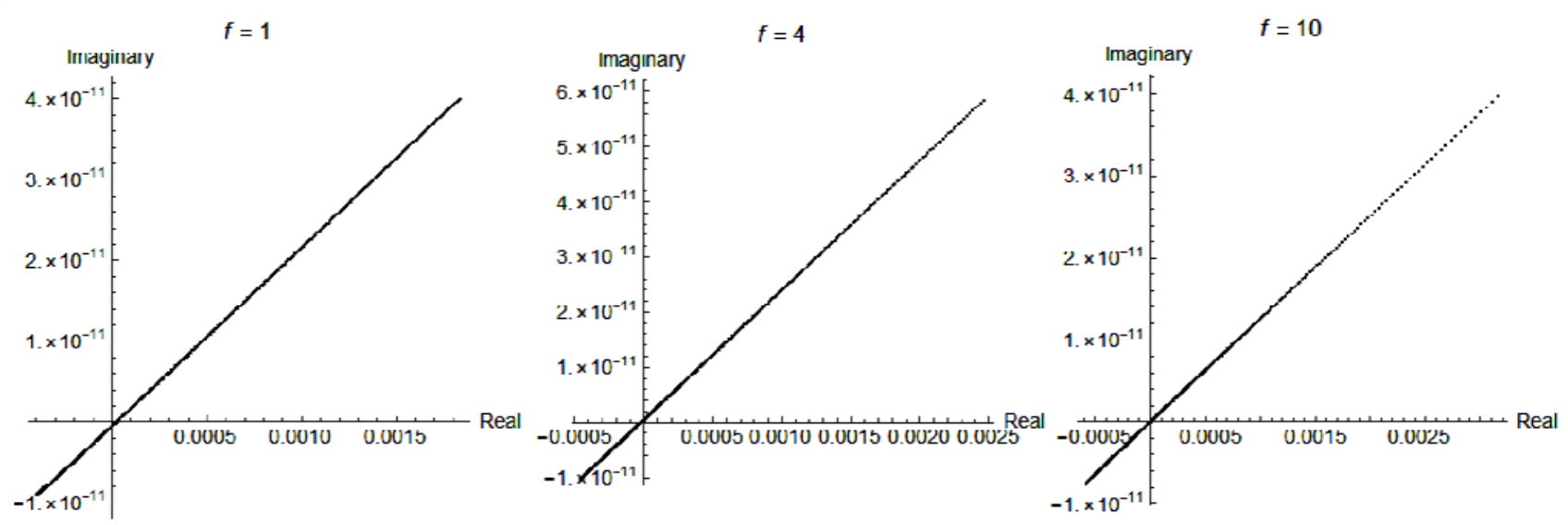

Fig.11. Complex solution for shear stress $\left(\sigma_{X Y}\right)$ across the thickness for the volume fraction exponent (f) and $a / h=4$.
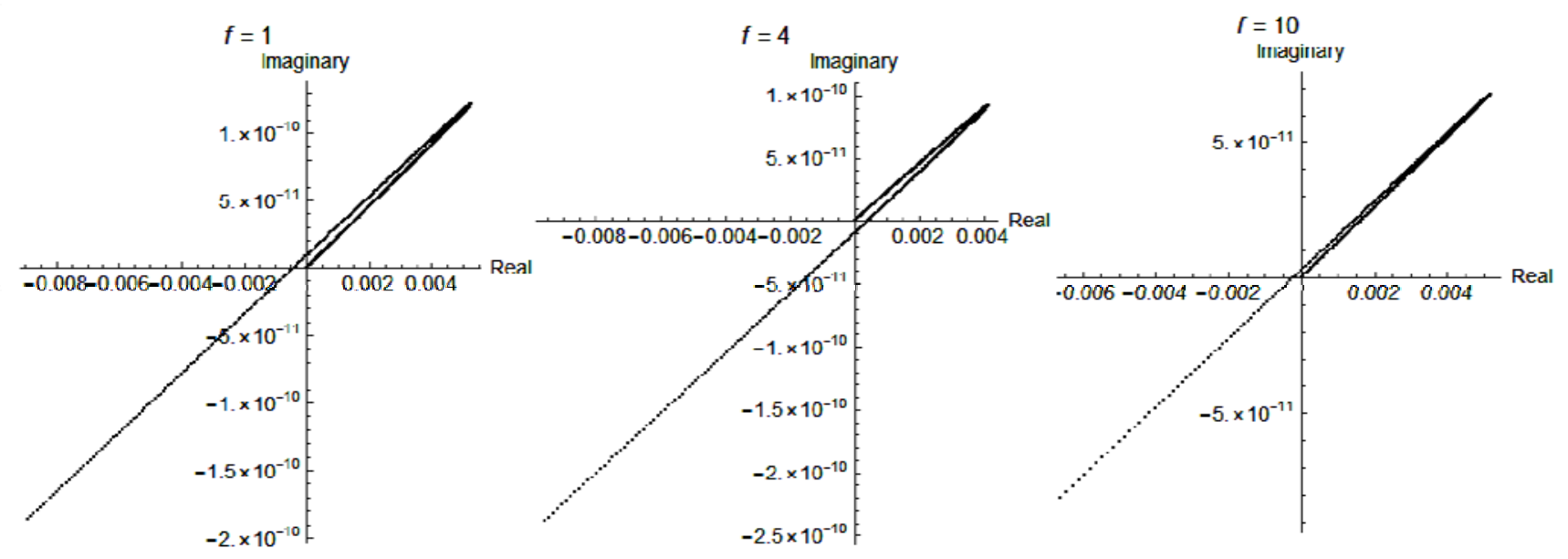

Fig.12. Complex solution for transverse shear stress $\left(\sigma_{X Z}\right.$ or $\left.\sigma_{Y Z}\right)$ across the thickness for the volume fraction exponent (f) and $a / h=4$. 
The following observations have been made for the functionally graded plate for the real part of the complex solution of stresses:

1) As the volume fraction exponent increases, the stress behaves linearly at the bottom of the plate and becomes nonlinear as it approaches towards the top of the plate as shown in Figs 13 and 15.

2) The transverse normal stress is approximately zero at the bottom of the plate and varies non-linearly as it approaches towards the top of the plate. Also, it can be seen that for the volume fraction exponent 4 , the stress generated across the thickness is least as shown in Fig.14.

3) The transverse shear stress varies parabollically along the thickness of the plate with maximum at $z=0$ and minimum at the top and bottom surfaces of the plate. Again, it can be seen that for the volume fraction exponent 4 , the transverse shear stress generated across the thickness is least as shown in Fig.16.

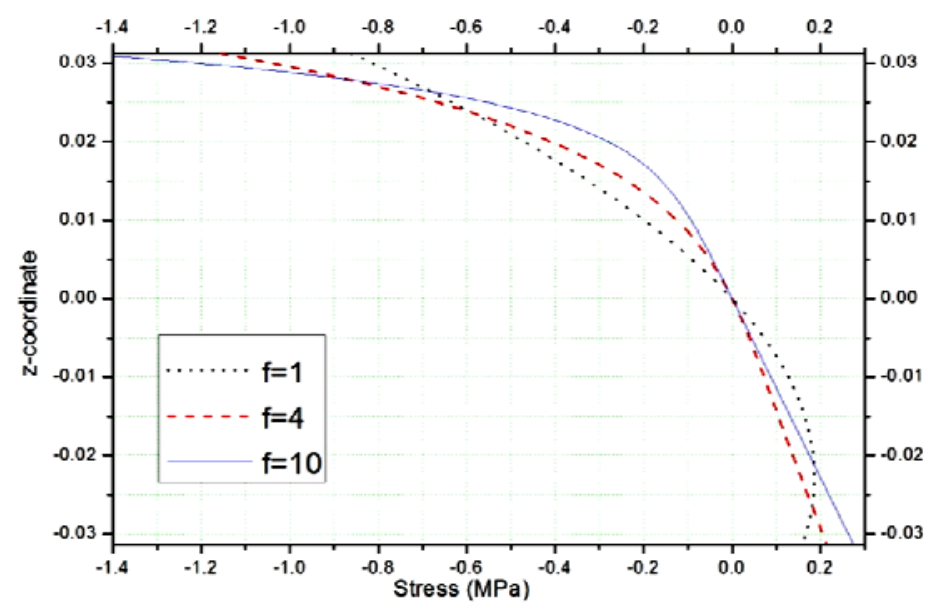

Fig.13. Real part of the solution for normal stress in the $X$ or $Y$-direction across the thickness for the volume fraction exponent (f) and $a / h=4$.

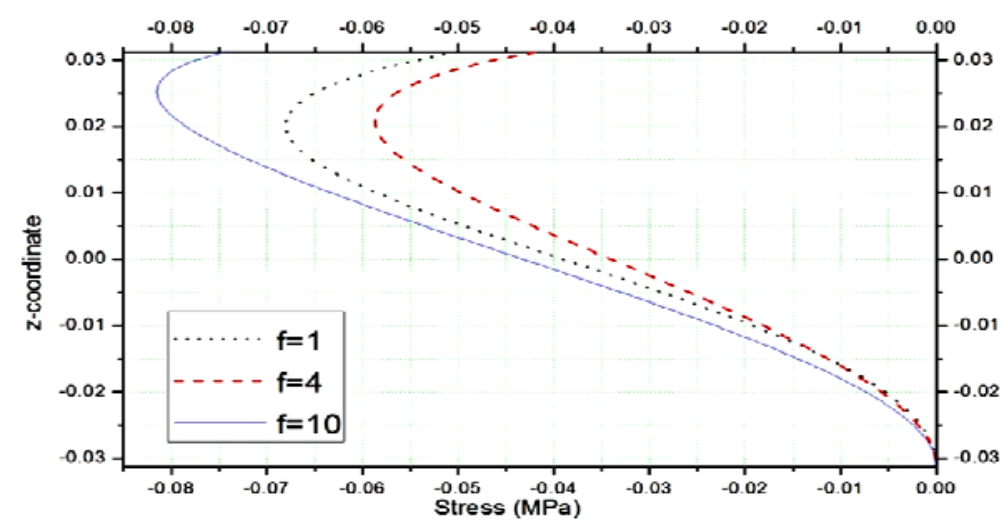

Fig.14. Real part of the solution for transverse normal stress in the Z-direction across the thickness for the volume fraction exponent (f) and $a / h=4$. 


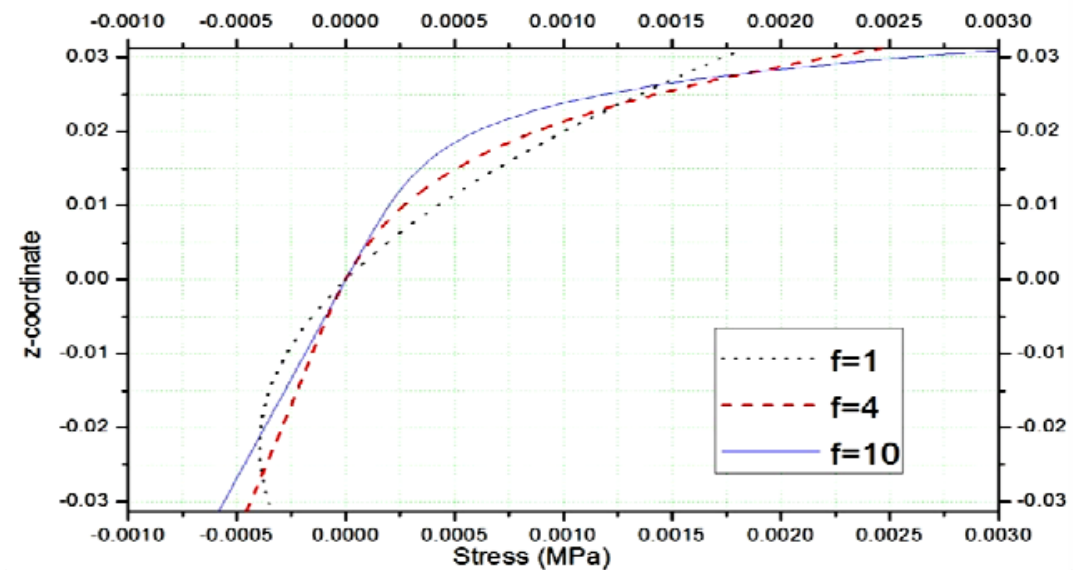

Fig.15. Real part of the solution for shear stress $\left(\sigma_{X Y}\right)$ across the thickness for the volume fraction exponent (f) and $a / h=4$.

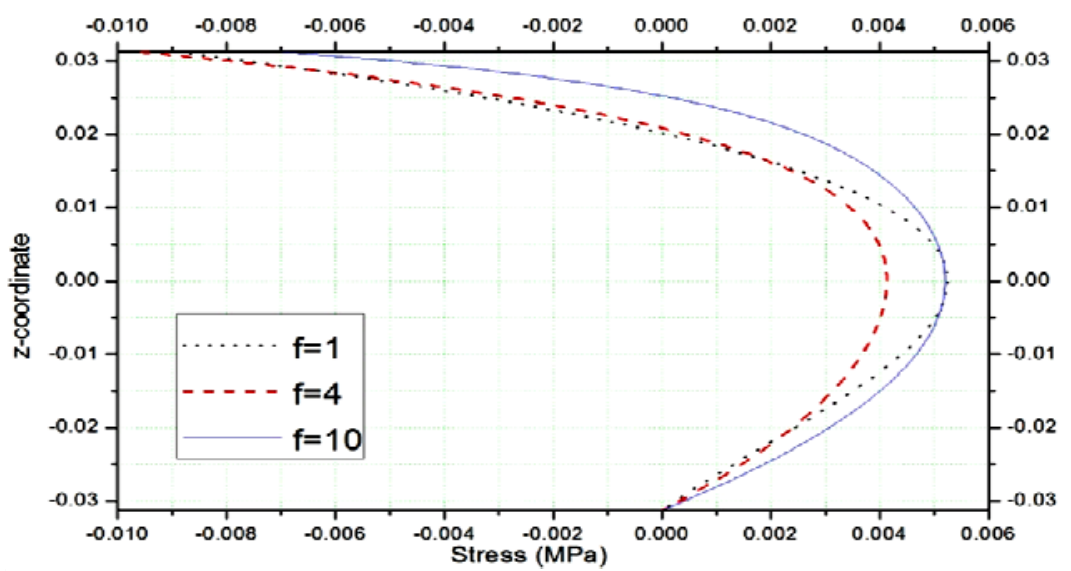

Fig.16. Real part of the solution for transverse shear stress $\left(\sigma_{X Z}\right.$ or $\left.\sigma_{Y Z}\right)$ across the thickness for the volume fraction exponent (f) and $a / h=4$.

\section{Conclusions}

In this study, an analytical solution to a functionally graded plate based on the two-dimensional nonlinear classical plate theory is carried out. The solution obtained using Navier's method is complex and hence a complex analysis is made in the present study. Non-linear bending and stress under a transverse uniform distributed load with diaphragm edge conditions are analyzed. The material property variation across the thickness according to simple power law is more effective for moderately thick $(a / h=5-10)$ and thick plates $(a / h<5)$. The complex nature of the solution also gives information about the stress distribution and indicates the severity of stress in the thickness direction. The stresses in a functionally graded material lie in between the stresses in metal and ceramic due to a reduction in stiffness property of a material. The shear stresses are more induced in thin plates rather than thick and moderately thick plates. Also, the stresses induced in pure ceramic and metal are equal, so for homogeneous materials, stresses are independent of elastic stiffnesses. The material with the volume fraction exponent 4 is subjected to much smaller transverse normal and shear stress in comparison to other volume fraction exponents. 


\section{Nomenclature}

$a, b$ - plate dimensions

$a / h$ - span-to-thickness ratio

$C$ - reduced stiffness of the plate

$E_{m}, E_{c}-$ modulus of elasticity for metal and ceramic material, respectively

$f$ - volume fraction exponent

$h$ - thickness of the plate

$N, M-$ stress and moment resultant

$u, v, w$-displacement of the plate in the $x$-, $y$ - and $z$-direction, respectively

$\bar{u}, \bar{v} \quad$ - in-plane displacement of the plate in the $x$ - and $y$-direction, respectively

$\bar{w}-$ transverse displacement of plate in the $z$-direction

$\bar{w} / h$ - dimensionless central deflection of the plate

$v$ - Poison's ratio of plate material

$\sigma_{X X}, \sigma_{Y Y}-$ normal stresses in the $x$ - and $y$-direction

$\sigma_{X Y}-$ shear stresses in the $x-y$ plane

$\sigma_{X Z}, \sigma_{Y Z}-$ transverse shear stresses in the $x-z$ and $y$-z plane, respectively

$\sigma_{Z Z}-$ transverse normal stresses

\section{References}

[1] Koizumi M. and Niino M. (1995): Overview of FGM research in Japan. - MRS Bulletin, vol.20, No.1, pp.1924.

[2] Mortensen A. and Suresh S. (1995): Functionally graded materials and metal-ceramic composites. - Part I: Processing, International Materials Reviews, vol.40, No.6, pp.239-265.

[3] Wang S.S. (1983): Fracture mechanics for delamination problems in composite materials.- Journal of Composite Materials, vol.17, No.3, pp.210-223.

[4] Niino M., Hirai T. and Watanabe R. (1987): The functionally gradient materials. - Journal of the Japan Society for Composite Materials, vol.13, pp.257-264.

[5] Report (1992) on: Fundamental study on relaxation of thermal stress for high temperature material by tailoring the graded structure. - Department of Science and Technology Agency.

[6] Marin L. (2005): Numerical solution of the Cauchy problem for steady-state heat transfer in two dimensional functionally graded materials. - International Journal of Solids Structures, vol.42, pp.4338-4351.

[7] Müller E., Drašar C., Schilz J. and Kaysser W.A. (2003): Functionally graded materials for sensor and energy applications. - Materials Science and Engineering: A, vol.362, pp.17-30.

[8] Niino M., Kisara K. and Mori M. (2005): Feasibility study of FGM technology in space solar power systems (SPSS). - Materials Science Forum, vol.492, pp.163-168.

[9] Levy S. (1942): Bending of rectangular plates with large deflections. -NACA Technology Note 846, pp.1-46.

[10] Kant T. and Swaminathan K. (2001): Analytical solutions for free vibration of laminated composite and sandwich plates based on a higher order refined theory. - Composite Structures, vol.53, pp.73-85.

[11] Huang M., Ma X.Q., Sakiyama T., Matuda H. and Morita C. (2005): Free vibration analysis of orthotropic rectangular plates with variable thickness and general boundary conditions. - Journal of Sound and Vibration, vol.288, No.4-5, pp.931-955.

[12] Ke L.L. and Wang Y.S. (2011): Size effect on dynamic stability of functionally graded microbeams based on a modified couple stress theory. - Composite Structures, vol.93, No.2, pp.342-350.

[13] Thai H.T. and Choi D.H. (2013): Size-dependent functionally graded Kirchhoff and Mindlin plate models based on a modified couple stress theory. - Composite Structures, vol.95, pp.142-153. 
[14] Thai H.T. and Kim S.E. (2013): A size-dependent functionally graded Reddy plate model based on a modified couple stress theory. - Composite Part B Engineering, vol.45, No.1, pp.1636-1645.

[15] Thai H.T. and T.P. Vo (2013): A size-dependent functionally graded sinusoidal plate model based on a modified couple stress theory. - Composite Structures, vol.96, pp.376-383.

[16] Thai H.T. and Vo T.P. (2013): A new sinusoidal shear deformation theory for bending, buckling, and vibration of functionally graded plates. - Applied Mathematical Modelling, vol.37, No.5, pp.3269-3281.

[17] Reddy B.S., Kumar J.S., Reddy C.E. and Kumar K.V. (2014): Static analysis of functionally graded plates using higher-order shear deformation theory- International Journal of Applied Science and Engineering vol. 17, no. April 2013, pp. 23-41.

[18] Kumar R. (2016): Meshless analysis of functionally graded plate with different algebraic shear deformation theories. - International Journal for Innovative Research in Science and Technology, vol.2, No.8, pp.106-111.

[19] Setoodeh A.R. and Shojaee M. (2016): Application of TW-DQ method to nonlinear free vibration analysis of FG carbon nanotube-reinforced composite quadrilateral plates. - Thin-Walled Structures, vol.108, pp.1-11.

[20] Neves A.M.A., Ferreira A.J.M., Carrera E., Roque C.M.C., Cinefra M., Jorge R.M.N. and Soares C.M.M. (2011): Bending of FGM plates by a sinusoidal plate formulation and collocation with radial basis functions. Mechanics Research Communications, vol.38, No.5, pp.368-371.

[21] Neves A.M.A., Ferreira A.J.M., Carrera E., Cinefra M., Roque C.M.C., Jorge R.M.N. and Soares C.M.M. (2012): A quasi-3D hyperbolic shear deformation theory for the static and free vibration analysis of functionally graded plates. - Composite Structures, vol.94, No.5, pp.1814-1825.

[22] Neves A.M.A., Ferreira A.J.M., Carrera E., Roque C.M.C., Cinefra M., Jorge R.M.N. and Soares C.M.M. (2012): A quasi-3D sinusoidal shear deformation theory for the static and free vibration analysis of functionally graded plates. - Composite Part B Engineering, vol.43, No.2, pp.711-725.

[23] Neves A.M.A., Ferreira A.J.M., Carrera E., Cinefra M., Roque C.M.C., Jorge R.M.N. and Soares C.M.M. (2013): Static, free vibration and buckling analysis of isotropic and sandwich functionally graded plates using a quasi-3D higher-order shear deformation theory and a meshless technique. - Composite Part B Engineering, vol.44, No.1, pp.657-674.

[24] Hassaine T., Tounsi A., Abbes E. and Bedia A. (2013): Analytical solution for bending analysis of functionally graded plates. - Scientia Iranian, vol.20, No.3, pp.516-523.

[25] Kulkarni K., Singh B.N. and Maiti D.K. (2015): Analytical solution for bending and buckling analysis of functionally graded plates using inverse trigonometric shear deformation theory. - Composite Structures, vol.134, pp.147-157.

Received: June 6, 2017

Revised: June 20, 2018 